\title{
Ein Blick unter die Motorhaube des Zentralvorstandes
}

\author{
Jürg Unger-Köppel \\ Dr. med., Mitglied des FMH-Zentralvorstandes, Departementsverantwortlicher Stationäre Versorgung und Tarife
}

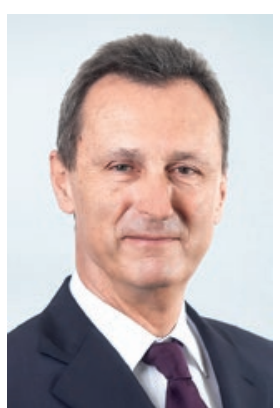

Ältere Ärzte treffen sich und diskutieren: «Im ZV braucht es zwingend einen Chirurgen» - «Ja, und auch einen Internisten" - "Aber ganz sicher jemanden aus der Romandie.» Würde die Diskussion wohl anders verlaufen, wenn junge Ärztinnen dieses Thema diskutierten? Vielleicht hiesse es dann: «Es muss unbedingt jemand in den $\mathrm{ZV}$, die sich für die Vereinbarkeit von Familie und Beruf einsetzt» - «Ja, und für die Einhaltung der Arbeitszeiten" - "Und bitte endlich mehr Frauen in den ZV» - «Und Einsatz für beste Weiterbildungsbedingungen und für minimale Administration.» Und wie liefe das Gespräch bei jungen Ärzten und älteren Ärztinnen?

Die Aussensicht auf ein Gremium, die damit verbundenen Anliegen und Wünsche sind das eine. Die Innensicht jener, die Mitglied im Gremium sind, ist oft eine andere. Schauen wir deshalb zusammen etwas «unter die Motorhaube» des aktuellen Zentralvorstandes.

Was braucht es im ZV? Und wie liefe das Gespräch darüber wohl unter jungen Ärztinnen?

Die medizinische Grundausbildung spielte in unseren Diskussionen nur insofern eine Rolle, als die Vernetzung mit den verschiedenen Fachgruppen innerhalb der FMH unterschiedlich war. Aber alle diskutierten immer für die gesamte Ärzteschaft und versuchten Lösungen zu finden, die möglichst vielen der mehr als 42000 Mitglieder gerecht würden. Wenn bei einer Fragestellung die Meinung einer wichtigen Fachgruppe im Gremium nicht klar war, so haben wir stets bei den entsprechenden Kolleginnen und Kollegen nachgefragt. Damit (muss)/möchte ich der eingangs zitierten Meinung der älteren Ärzte widersprechen: Wichtig ist nicht Chirurgie, innere Medizin, Psychiatrie oder pädiatrische Neuroradiologie, sondern die Fähigkeit, die Interessen der ganzen Ärzteschaft zu erkennen und angemessen zu vertreten. Zustimmen möchte ich ihnen dagegen, was die Vertretung der Sprachregionen anbelangt, denn der gesundheitspolitische Alltag zwischen Lac Léman, Bodensee und Lago di Lugano ist oft sehr unterschiedlich. Die verschiedenen Realitäten zu erkennen ist für die Arbeit des Zentralvorstandes wesentlich. Einen weiteren wichtigen Unterschied bilden die Herausforderungen der Ärzteschaft im Spital und in der Praxis. Auch diese zwei unterschiedlichen Erfahrungswelten müssen unbedingt vertreten sein, damit die Diskussionen zu ausgeglichenen Ergebnissen führen können. Nicht zuletzt sind auch für die Mitarbeitenden der FMH das Wissen und die Erfahrung der Zentralvorstandsmitglieder zu Organisation und Prozessen für die sie betreffenden Führungsentscheide sehr wichtig.

Wie wurde mit den Bedürfnissen der jüngeren Generation umgegangen in einem Gremium von Ü50? Ein Mitglied des Gremiums sorgte für den permanenten Informationsaustausch mit dem VSAO, und die übrigen Mitglieder bezogen je nach Thema den VSAO eng in die Entscheidungsfindung mit ein. Mit einer starken Frau im Gremium und gemeinsam mit der Generalsekretärin hatten spezifische Anliegen der Frauen deutliche Stimmen.

Viele nehmen den Präsidenten der FMH nur über seine Aussenwirkung wahr. Aber die Art, wie er die zahlreichen und mit vielen Themen befrachteten Sitzungen des Zentralvorstandes leitet, ist entscheidend für das Verhältnis der Mitglieder des Gremiums zu- und untereinander und in Bezug auf die erzielten Diskussionsresultate. Es ist sicher ein Zeichen der hervorragenden Arbeit des abtretenden Präsidenten, dass auch bei eingangs grossen Meinungsunterschieden nach stattgehabter Diskussion vielfach einstimmige Resultate mit guten Kompromissen resultierten. Unter seiner Führung arbeiteten alle viel und sehr engagiert für ihr Amt - und das ohne irgendwelche Leistungsanreize oder Boni. Gewiss war dabei neben der Arbeit des Präsidenten ein ganz wesentlicher Erfolgsfaktor, dass die einzelnen Mitglieder des Zentralvorstandes ihre Aufgabe als Teamplayer und nicht als Selbstdarsteller verstanden.

So sei mir eine persönliche Feststellung zum Schluss erlaubt: In 20 Jahren in Geschäftsleitungen und Verwaltungsräten habe ich nie eine so angenehme und konstruktiv lösungsorientierte Zusammenarbeit erlebt wie in diesem Zentralvorstand. 